\title{
About A Rare Case: Isolated Bilateral Multi-Ligament Knee Injury
}

\section{Dr. Azzelarab Bennis ${ }^{1 *}$, Dr. Jalal Youssef ${ }^{2}$, Dr. Naoufal Elghoul ${ }^{3}$, Dr. Mohammed Benchakroun ${ }^{4}$, Dr. Mansour Tanane ${ }^{5}$,} Dr. Abdeloihab Jaafar ${ }^{6}$, Dr. Ali Zine ${ }^{7}$, Dr. Salim Bouabid ${ }^{8}$

\begin{abstract}
$1,4,5,6,7,8$ Professor in Orthopedic Surgery and Traumatology, ${ }^{2,3}$ Orthopedic Surgery and Traumatology, Department of Orthopedic Surgery and Traumatology, Military Hospital Mohammed V (HMIMV), Faculty of Medicine and Pharmacy, Mohammed V University of Rabat, Morocco
\end{abstract}

DOI: $10.36347 /$ sjmcr.2020.v08i06.002

| Received: 20.05.2020 | Accepted: 02.06.2020 | Published: 06.06.2020

*Corresponding author: Bennis Azzelarab

Abstract

Multi-ligament knee injuries (MLKI) are rare but severe injuries that can threaten limb viability, accounting for less than $0.2 \%$ of all orthopedic injuries. There is a paucity of high-quality evidence upon which to base the management of MLKI, and treatment strategies for MLIK are challenging for most orthopedic surgeons. In this paper, we present a case of isolated bilateral multi-ligament knee injuries to describe our algorithm treatment for such injuries.

Keywords: Multi-ligament knee injury, algorithm treatment, Rehabilitation.

Copyright @ 2020: This is an open-access article distributed under the terms of the Creative Commons Attribution license which permits unrestricted use, distribution, and reproduction in any medium for non-commercial use (NonCommercial, or CC-BY-NC) provided the original author and source are credited.

\section{INTRODUCTION}

A Multi-ligament knee injury (MLKI) is commonly defined as disruption to at least two of the four major knee ligaments structures: anterior cruciate ligament (ACL), posterior cruciate ligament (PCL), medial collateral ligament (MCL), posteromedial corner, lateral collateral ligament (LCL), or posterolateral corner. The incidence of these serious injuries is less than $0.2 \%$ of all orthopedics traumas [1, $2]$. They are often associated with popliteal artery injury (4.8 to $65 \%$ ) [3], common fibular nerve injury (20\%) [4], acute compartment syndrome, deep venous thrombosis, or fracture (53\%) [5]. However, isolated traumatic bilateral MLKI following high-velocity trauma are even rare injuries, and they are only reported in a few case reports $(5 \%$ of all patients with ligaments knee injuries) [6]. The major challenge regarding MLKI is making a treatment algorithm to achieve the best functional outcome possible with a minimum of complications [7]. In the following text, we present a case of bilateral multi-ligament knee injuries, with two main concerns: making the diagnosis of this potentially limb-threatening injury, and describing our treatment algorithm.

\section{Case Report}

26 years old male serving soldier was admitted to the emergency department after being hit by a car, with frontal impact at both knees. He received initial care at other local hospital for recognized bilateral knee dislocation, with successfully closed reduction and cast- bracing immobilization for both knees, referred 6h later to our department for specific management. In the initial examination, He had on his left knee a deformity, swelling, and bruising. On his right knee, he had just abrasion and swelling. The ligament stability could not be evaluated due to pain. The neurologic examination findings were normal, and there were no signs of vascular impairment.

The anteroposterior and frontal X-rays of both knees confirmed successful closed reduction, with no fracture or bone avulsion (figure1). So, we performed a MRI scan of both knees that showed: on the right knee, a tear of LCL and MCL, rupture of the posterolateral capsule, torn popliteus muscle, complete PCL tear, partial tear of the posterolateral bundle of $\mathrm{AC}$; on the left knee, a complete rupture of both ACL and PCL, with rupture of the MCL (figure 2). These injuries were classified as KD IV at the right knee and KDIII-M at the left one according to the modified schenck classification [8].

After receiving informed consent, the surgical staff decided to manage these injuries in two stages: the first step was consisted of confirming the imaging data by an examination under general anesthesia and stabilize the left knee; the second step was aimed to manage the right knee injuries.

In the operating room, the patient was positioned supine on the table. Under general 
anesthesia, physical exam with stress radiographs found: (figure 3,4 ).

- For the right knee: significant laxity during varus and valgus stress testing at both $0^{\circ}$ and $30^{\circ}$ of knee flexion. The Lachman test, anterior drawer test, and pivot shift test were positive, just as posterior drawer test at 90 degrees of knee flexion.

- On the left side: The anterior drawer test, the Lachman test and posterior drawer test were positive with laxity during valgus stress testing just at $30^{\circ}$ of knee flexion.

To control the posterior subluxation and maintain anatomical reduction of the left knee, olecranization of the patella was decided. The Steinmann pin was placed for six weeks (figure 5). Full mobility between 0 degrees and 90 degrees was maintained.

Six days later, the patient was readmitted in the operating room. He was placed in a supine position with a roll placed under the opposite hip. A tourniquet was applied on the proximal right thigh. Firstly, a medial longitudinal incision was made, followed by a careful dissection of the subcutaneous tissue, then the medial retinaculum and capsule were torn. The MCL was completely detached from the femoral footprint (figure 6). The latter was reattached using a $5 \mathrm{~mm}$ corkscrew, reinforced by a staple. The ruptured posteromedial capsule was closed with non-absorbable sutures. This surgical approach allowed harvesting the semitendinosus tendon. Second, a curved incision centered proximally on the lateral epicondyle, and distally it follows a $5 \mathrm{~cm}$ arc around Gerdy's tubercle was made. The subcutaneous tissue was then dissected, and the long and short heads of the biceps femoris were exposed. The common peronea nerve was intact. After the neurolysis, a vertical arthrotomy was performed anteriorly to the popliteus tendon. The intraoperative exploration confirmed the MRI results. Reconstruction of the PCL was performed using a semitendinosus tendon graft fixed by two interference screws. The popliteus tendon was repaired and renforced by a staple, the posterolateral corner with nonabsorbable sutures placed in the biceps femoris, popliteofibular ligament, and lateral collateral ligament and suture anchors in the posterolateral and lateral tibia (figure 7,8 ). Six months later, Arthroscopic ACL reconstruction for the right knee was performed using Achilles tendon allograft.

Three weeks later, the patient was placed in right knee braced locked in full extension without weight-bearing. During this time, early passive rehabilitation was started using the continuous passive motion device for the knee for $45 \mathrm{~min}$ per day, with a goal of full extension and $90^{\circ}$ of passive knee flexion at three weeks, in junction with isometric quadriceps exercises. In parallel, elevation and cryotherapy were used to control postoperative edema and pain. After three weeks post-operatively, with the brace locked in full extension, partial weight bearing was initiated, and increased until full weight-bearing is resumed. During this time, rehabilitation continued to focus on quadriceps stretching, patellar mobilization, overpressure into knee extension, and the use of a mechanical passive knee flexion device. At twelve weeks post-operatively, the patient's flexion was $130^{\circ}$, while extension changed from his 1-week post-surgery measurement $\left(2^{\circ}-0^{\circ}\right)$. For the Left knee, Physiotherapy started immediately and early weight bearing was allowed with a posterior splint. At one year follow up (figure 9), the range of motion in the right knee was $0^{\circ}$ to $135^{\circ}$, and in the left one is $0^{\circ}$ to $115^{\circ}$. The left knee has a positive anterior drawer (grade I) and positive posterior drawer (grade I). For the right one, the anterior drawer test, Lachman test and posterior drawer test were negative. Functionally, He had no limp and walked without support. He could negotiate stairs without problems; however, he still reports mild pain in the posterior aspect of the left knee at end range flexion, rated as 3 to 4 on a 0 to 10 pain scale. The lysholm score was $96 \%$ for the right knee against $85 \%$ for the left one. At his 7-months postoperative he returned to work. At the last follow up Radiographs at that time demonstrated Osteoarthritis of the internal and external femorotibial compartment grading II. However, the patient was satisfied with his function and stability and felt they are adequate to perform job related activities and activities of daily living.

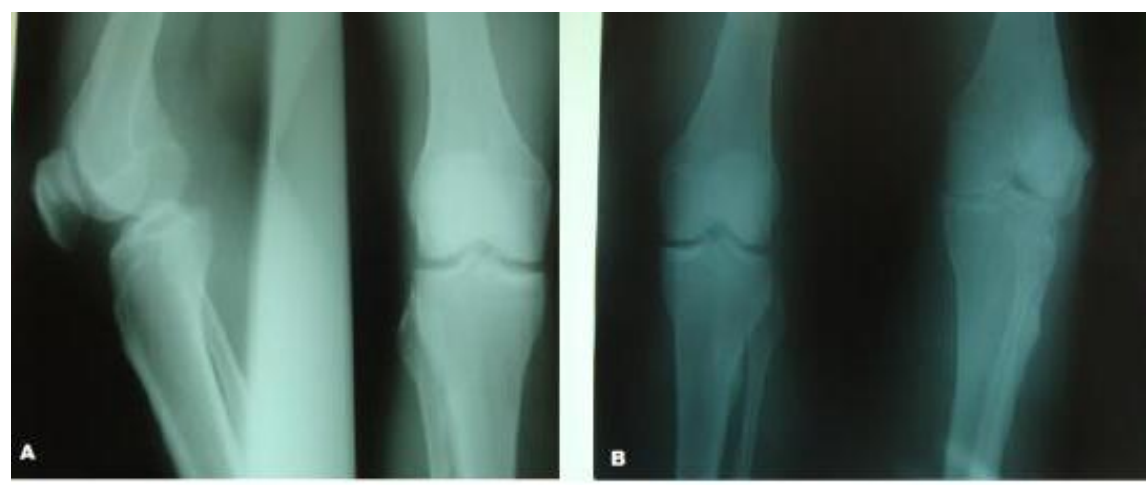

Fig-1: Anteroposterior and lateral radiographic views of the both knee showing successful reduction. A. left knee B. right knee 

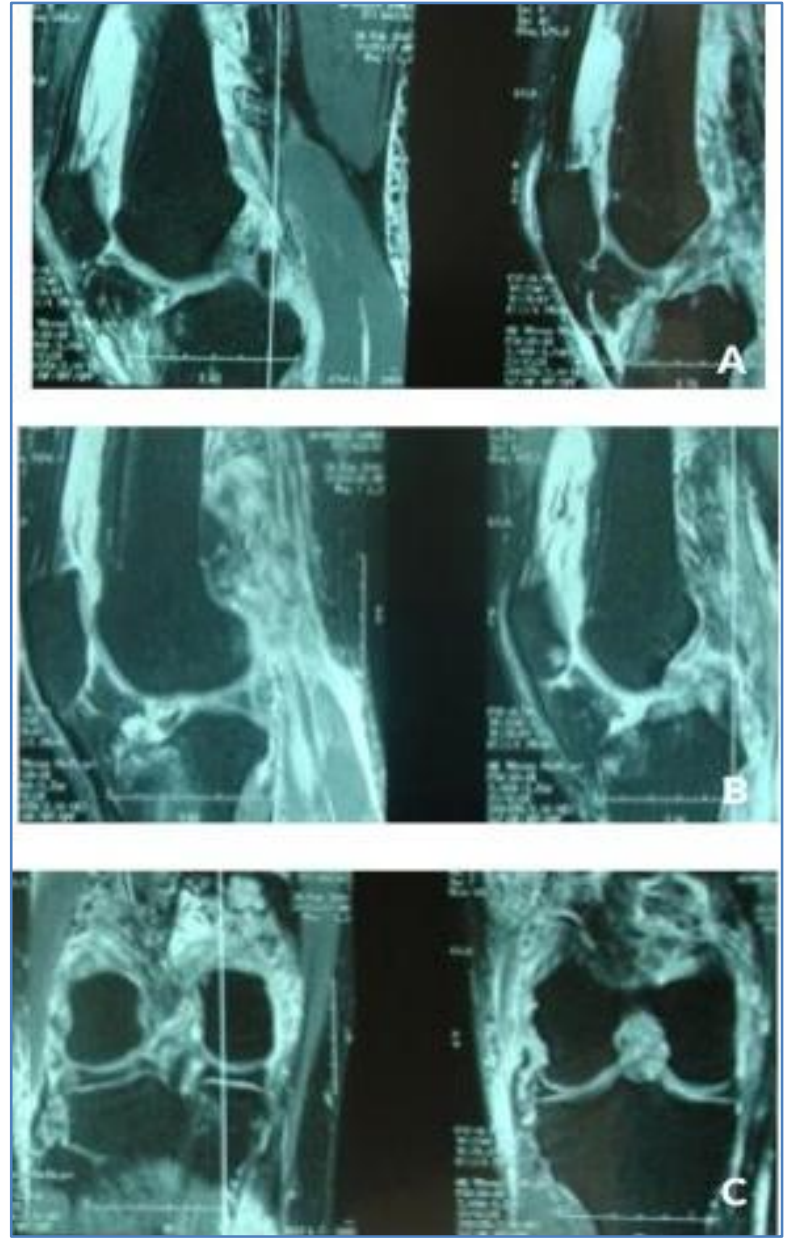

Fig-2: Findings MR imaging for both knees: A. Sagittal PDW left knee MR image demonstrating a complete tear of the PCL and ACLB. Sagittal PDW right knee MR image demonstrating a complete tear of the PCL and ACLC Coronal PDW-SPAIR right knee MR image demonstrating MCL and LCL sprain

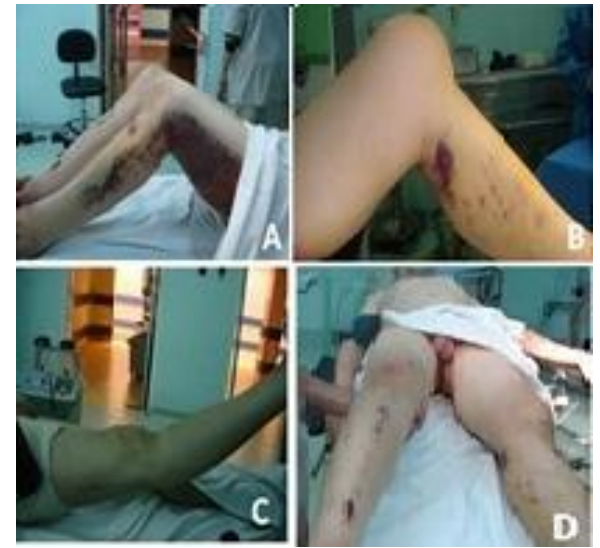

Fig-3: (A, B, C, D): physical exam under general anesthesia

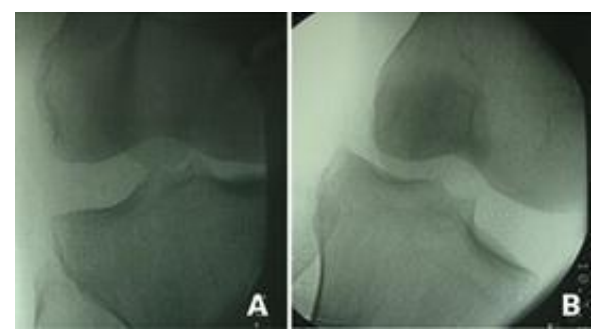

Fig-4: Stress radiographs for the right knee. A. a varus stress radiograph of an LCL injury $B$. a valgus stress radiograph of an MCL

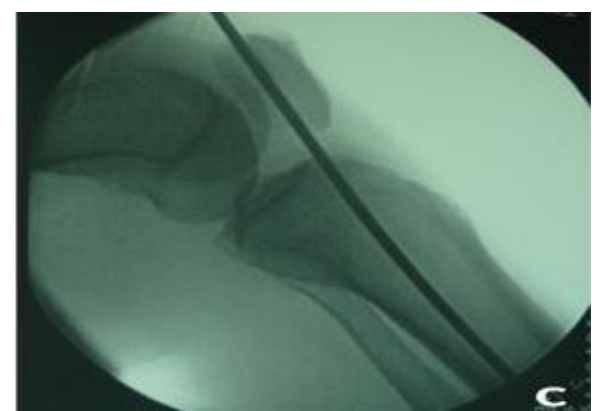

Fig-5: Olecranization of the patella for the left knee
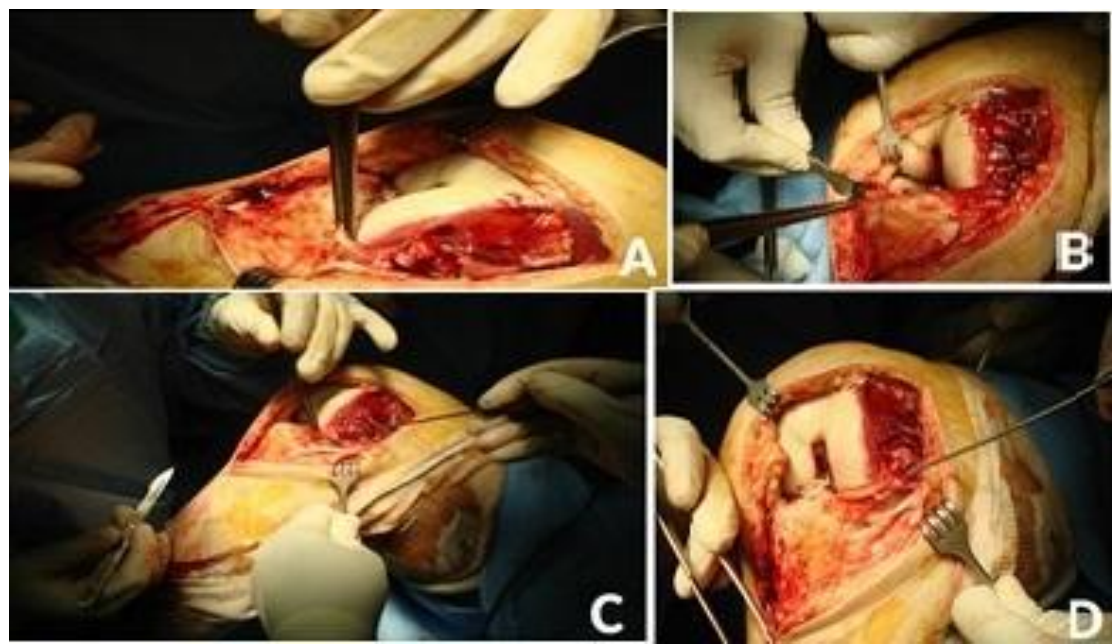

Fig-6 (A, B, C, D): Intraoperative clinical photographs of medial approach: the medial retinaculum and capsule were torn. The MCL was completely detached from the femoral footprint. The latter was repaired and reinforced by a staple, just as the medial retinaculum and capsule 

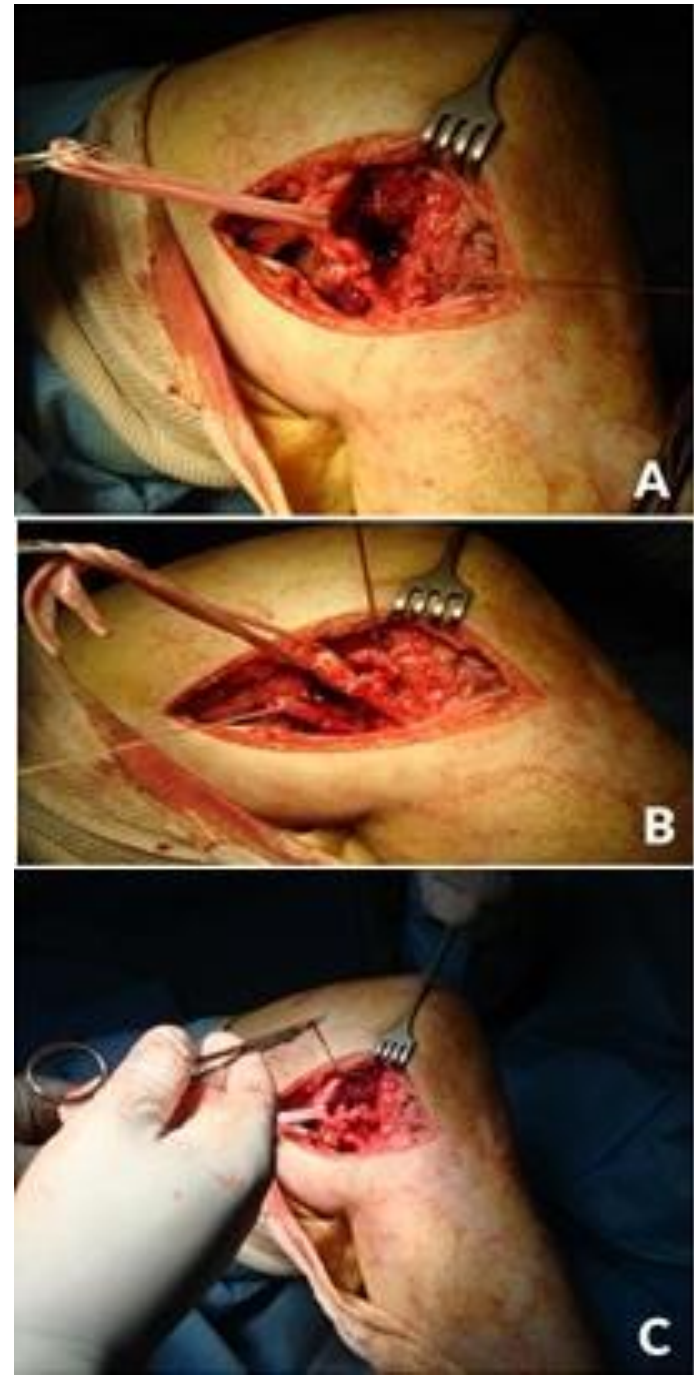

Fig-7 (A,B,C): Intraoperative clinical photographs of lateral approach : Primary repair of an acute posterolateral corner injury with nonabsorbable sutures placed in the biceps femoris, popliteofibular ligament, and lateral collateral ligament and suture anchors in the posterolateral and lateral tibia

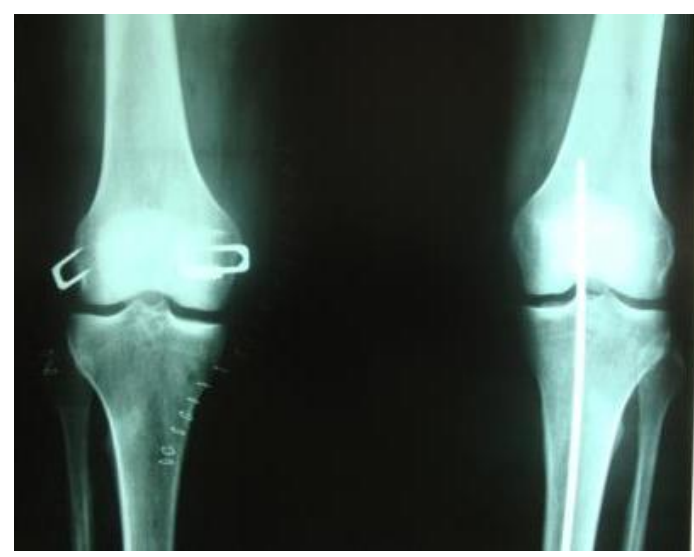

Fig-8: Radiographs control of the knees
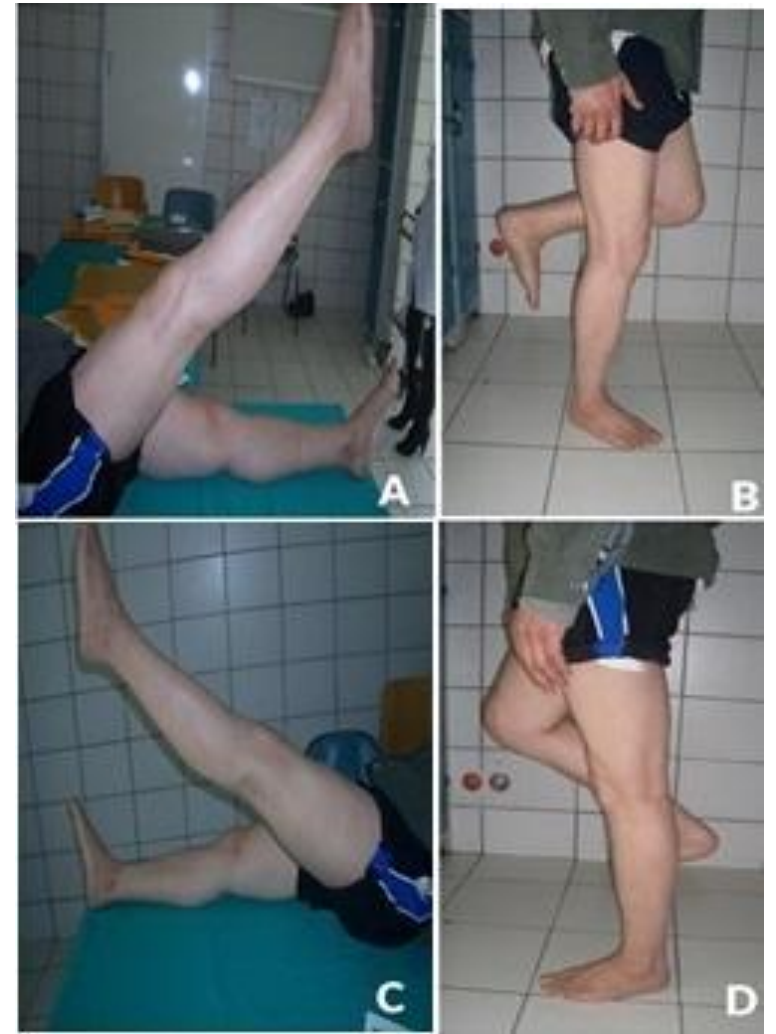

Fig-9: Physical exam at one year follow up

\section{DISCUSSION}

Multiligamentous knee injuries are complex extremity injuries, with a reported incidence of less than $0.2 \%$ of all orthopedics injuries. Most commonly, MLKI were associated with neurovascular injuries and concomitant fractures of the involved limb. However, isolated bilateral forms are rare [5]. These injuries are typically the result of severe trauma [6]. In this condition, various forces can be applied to the knee including translation (anterior and posterior), angulation (varus and valgus), rotation (internal and external), hyperextension, axial load, and direct blow. Usually, MLKI occurs as a result of two or more forces exerted across either a flexed or extended joint. In our case, the mechanism was direct trauma onto an extended knee, with the combined application of a varus force.

Initial evaluation and management of such injury represent main concerns which require a high level of vigilance by the surgeons. Thus, care must be taken to identify fractures and dislocations, and the latter must be reduced as soon as possible. The vascular status of the limb must be determined before and after reduction. The incidence of an associated vascular injury reported in the literature ranges from 11 to $64 \%$ [3]. If the limb is ischemic, emergent surgical exploration and revascularisation are required. Following high-energy trauma care, MRI proved useful in the establishment of the diagnosis and for preoperative planning. 
Management of bilateral MLKI represents a special challenge for orthopedic surgeons. The decisions of operative versus nonoperative management, timing of surgery, repair versus reconstruction, use of allograft versus autograft, and rehabilitation protocols remain controversial.

Levy et al. [9] performed a systematic review of all studies comparing operative and non-operative treatment of MLKI up to August 2007. He found that patients receiving operative treatment returned to work earlier than those that where managed non-operatively (72\% versus $52 \%$ ). Surgically treated patients could also return to playing sport earlier than their nonoperatively managed counterparts (29\% versus $10 \%$ ). However, the difference of the range of motion between the groups failed to reach statistical significance $\left(126^{\circ}\right.$ versus $123^{\circ}$ ).

Regarding the optimal timing of surgery, Mook et al. [10] performed a systematic review of literature up to 2008, aiming to determine whether early or late surgery was associated with better outcomes. He found that acute treatment of knee dislocation led to increased anterior instability compared with chronic treatment. Furthermore, this study demonstrated that acute treatment is more likely to result in flexion loss of more than $10^{\circ}$ versus chronic treatment.

A wide variety of surgical techniques has been described for the treatment of acute MLKI [9, 10]. In this case, our propozed algorithm attempts to balance the restoration of stability with maintenance of function through the emergence of operative (repair and reconstruction) and nonoperative (olecranization). After a thorough search of the literature, we were unable to find reports with the same attitude to manage isolated bilateral MLKI. This practice has the advantage of not requiring specialized instrumentation, simple and quick to use in the treatment of these rare and complex injuries, with the successful outcomes.

Early rehabilitation is advocated by most of the authors. Whether the treatment is surgical or conservative, the rehabilitation progresses slowly into strengthening, gait and balancing activities and individual patients will progress at different rates depending on pre-injury function, the extent of the injury, and the commitment to the rehabilitation program [10].

\section{CONCLUSION}

Bilateral multi-ligament knee injuries are heterogenous and demand individualized treatment. In addition to the complexity of the injury, factors such as the timing and type of surgery are also crucial to patient outcomes. In this case, with isolated bilateral multiligament knee injury, the authors concluded that mixture of olecranization of patella and ligaments reconstruction can be successful and allow managing these complex knee injuries.

\section{REFERENCES}

1. Kennedy JC. Complete dislocation of the knee joint. J Bone Joint Surg Am. 1963; 45:889-904.

2. Hoover NW. Injuries of the popliteal artery associated with fractures and dislocations. Surg Clin North Am 1961; 41:1099-112.

3. McCoy GF, Hannon DG, Barr RJ, Templeton J. Vascular injury associated with low velocity dislocations of the knee. J Bone Joint Surg $\mathrm{Br}$ 1987; 69(2): 285-287

4. Monahan TJ. Management of acute and chronic nerve injuries in the multiple ligament injured knee. Sports Med Arthrosc. 2001; 9(3): 227-238

5. Stannard JP, Covey DC, Volgas D. Soft tissue injury of the knee after tibial plateau fractures. J Knee Surg. 2010; 23(4): 187-192.

6. Wascher DC, Dvirnak PC, DeCoster TA. Knee dislocation: initial assessment and implications for treatment. J Orthop Trauma. 1997;11: 525-9.

7. Levitsky KA, Berger A, Nicholas GG, Vernick CG, Wilber JH, Scagliotti CJ. Bilateral open dislocation of the knee joint. A case report. J Bone Joint Surg Am. 1988; 70(9):1407-1409

8. Schenck R.C. The dislocated knee. Instr Course Lect. 1994 ; 43: 127-136

9. Levy BA, Dajani KA, Whelan DB. Decision Making in the Multiligament-Injured Knee: An Evidence-Based Systematic Review. Arthroscopy. 2009; 25(4): 430-8

10. Mook WR, Miller MD, Diduch DR, Hertel J, Boachie-Adjei Y, Hart JM. Multiple-ligament knee injuries: a systematic review of the timing of operative intervention and postoperative rehabilitation. J Bone Joint Surg Am. 2009; 91(12): 2946-57. 